\title{
Adaptive Mesh Refinement and Domain Decomposition: A Framework to Study Multi-physical and Multi-scale Phenomena. First Application to Reacting Gas Flows
}

\author{
J. Ryan \\ ONERA,B.P. 72, 92322 Châtillon cedex, France \\ ryan@onera.fr
}

\begin{abstract}
The aim of this work is to extend the Adaptive Mesh Refinement (AMR) technique. AMR is an ideal platform for coupling different discretisations and models, as each grid level can use a different solver. Compact conditions have to be defined and these are problems which are at the core of Domain Decomposition methods. An application to a planar shock interacting with a circular diffusion H2-air flame is presented.
\end{abstract}

\section{Introduction}

The complexity of combustion and the subsequent difficulty of describing the intricate coupling between the many physical phenomena due to the presence of multiple length scales and the large number of species involved still limit the impact of numerical studies. One of the themes which is presented here is to extend the Adaptive Mesh Refinement (AMR) technique initially written for a finite volume algorithm to finite differences in order to integrate a 3D combustion solver. One of the main problems with AMR is the connection between coarse and fine patches. Domain decomposition methods can help to improve these. The final aim of our work is the coupling of various combustion solvers from simplified combustion model on a coarse grid to high precision DNS Finite Difference reacting gas model.

\section{Adaptive Mesh Refinement}

The locally adaptive mesh refinement method written at Onera by M. Borrel et al [2, originally developed by Berger and Oliger [1] and many other authors, uses a sequence of nested levels of refined structured grids (patches), on which different solvers on each level can be applied. These patches are built around cells that have been flagged with a problem dependant sensor, with a grouping/clustering technique.

The system of overlaid grids with successively finer spacing in time and in space needs the following operators to couple the different levels Prolongation operator 
(P), Restriction operator (R), Fine coarse boundary treatment (FC), Fine fine boundary treatment $(\mathbf{F F})$.

The first operator (P) usually consists in retrieving values from former patches and interpolating the missing values. The second operator ( $(\mathbf{R})$ )replaces coarse values by the overlying newly computed fine values. The last operator $(\mathbf{F F})$, as the scheme is entirely time explicit, is only coding work as it consists in retrieving computed values from neighbouring patches. Instabilities arise from the choice of the third operator (FC) . In the original Finite Volume method, fine coarse boundary treatments are dealt with by creating around the fine patch a set of fictitious points with values interpolated from the coarse grid. Fluxes between fine and coarse interfaces are readjusted after computation. Applied to high order finite difference discretisation, replacing interpolated values by values such that fine and coarse gradients were equal proved to be stable in all considered cases.

\section{$3 \quad \mathrm{AMR}$ and Combustion}

Thévenin's two-dimensional studies $[3$ have shown the necessity for a high precision solver (6th order in space and 4th order in time) with a large number of points. For three dimensional cases, this becomes quite prohibitive. Only locally adaptive and refined grids can resolve this impediment, especially as cases to be studied show locality of structures. To reduce even more computation costs , and as complex chemistry modeling is not necessary every where and all the time, a lower order algorithm MAJIC has been tested and integrated within the AMR platform. This method can run on coarse grids and detect areas that need to be refined in terms of geometry and model, and if necessary, a higher order solver can be used locally .

\subsection{MAJIC}

MAJIC is a 3D Finite Difference discretization code with a simplified combustion model developed by G. Billet [4. MAJIC uses a time-splitting method . The 3-D finite difference operator is split into a product of simpler explicit operators. The convective terms are solved by a $A U S M^{+}-M U S C L-$ triad approach ( [ $[$ ]). The "double flux model" presented in [6] is used to preserve pressure and velocity across the contact discontinuities. All the diffusion and dissipation terms are solved with a centered second-order scheme.

\subsection{Planar Shock Interacting with a Circular Diffusion H2-Air Flame}

A circular diffusion flame moving in a supersonic air flow $(M=2$.) interacts with a steady planar shock. The bubble contains a H2-N2 mixture $\left(Y_{H_{2}}=\right.$ $\left.0.233, Y_{N_{2}}=0.767\right)$ at temperature $T=300 \mathrm{~K}$ Upstream, the temperature of 


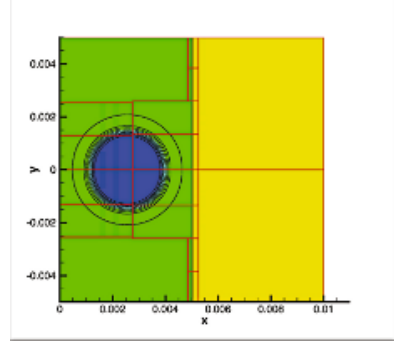

Fig. 1. Before the shock

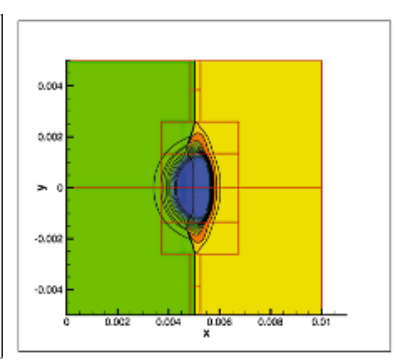

Fig. 2. Through the shock

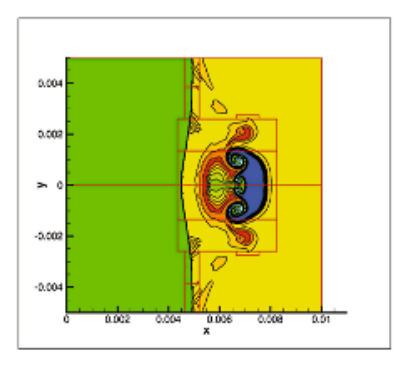

Fig. 3. After the shock

the ambient air is $1500 \mathrm{~K}$ and the pressure is uniform $(p=1$ bar $)$. This interaction sets up two counter-rotating vortices that are going to stretch the flame and enhance the total burning velocity. A detailed chemistry (9 species and 38 reactions) and detailed transport properties are used.

On figures (11213), are shown temperature evolution through the shock and the attached patches.

\section{AMR and Coupling}

AMR is an ideal platform for coupling different discretizations and models, as each grid level can use a different solver. In the classical AMR technique, fictitious points and interpolated values are created to allow the fine grid to compute the needed derivatives. To extend this to high order schemes, compact conditions have to be defined and these are problems which are at the core of Domain Decomposition methods.

\subsection{Artificial Boundary Conditions}

J. Gander, L. Halpern and F. Nataf in [5] developed optimal transmission conditions for space - time domain decompositions for wave propagation problems. When defining a finer patch, and using explicit time schemes, the time step to preserve the CFL number is reduced by the refinement factor. Fictitious points have thus to be defined at all the intermediate time steps. The space-time domain boundary conditions (Robin type conditions in time and space) mentioned above simplifies computation of these boundary values. From the underlying coarse domain, space and time derivatives can be computed at the boundaries of the fine patch, and combined to provide a boundary condition for the finer grid. Computation can then proceed in the fine patch. Fine values are then injected in the coarse grid.

First comparisons between the fictitious points technique and the time domain method ( Artificial boundary conditions, ABC) were performed in the case of the one dimensional unsteady convection diffusion of a Gaussian The ABC method gives better results for low order schemes, and are as good for a high order scheme. 


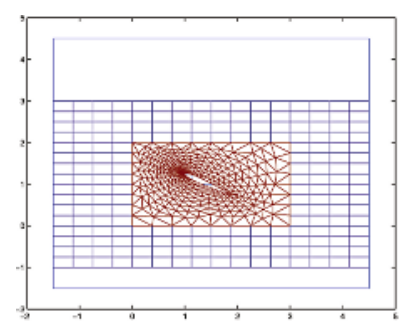

a) General mesh

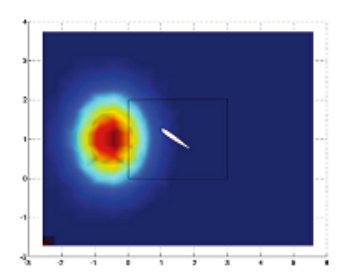

b) $\mathrm{T}=0 \delta t$

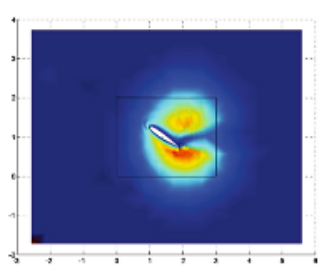

d) $\mathrm{T}=50 \delta t$

Fig. 4. Time evolution of a temperature blob round a NACA airfoil

Another test to validate the artificial boundary conditions was the $2 \mathrm{D}$ convection diffusion equation round a NACA airfoil. Two domains were used with a different solver :P1 finite elements, on the triangular mesh close to the airfoil, second order finite differences on the far-away field.

\section{Conclusion}

The AMR technique has shown to be valid in the case of different discretisations of the fluid equations. Future work will concern coupling different (simple to complex) modeling of these equations. The AMR-DDM technique can be extended to other fields of physics (solid mechanics, electro-magnetism, ...). The use of common tools cannot but be fruitful to develop multi-physics coupling.

\section{References}

1. Berger M. J., Oliger J. Adaptive Mesh Refinement for Hyperbolic Partial Differential Equations. J. Comput. Phys., 53, pp. 484-512, (1984).

2. Borrel M., Huré N. A MUSCL/DG AMR Method in 3D: A Multiblock Multigrid AMR Method in 3D using a MUSCL or a Discontinuous Galerkin Approach. Finite Volume for Complex Applications, Porquerolles, France, June 2002

3. Thévenin D., Gicquel O., Hilka M. and Darabiha N., Direct Numerical Simulation of turbulent premixed flames using intrinsic low-dimensional manifolds, Combustion Science and Technology ,149, pp.297-337 (1999)

4. Billet G. Vers l'extension de l'approche MUSCL-AUSM-triade à la LES et la DNS des écoulements compressibles visqueux. Application à la combustion instationnaire des flammes de diffusion avec chimie simplifiée. Rapport ONERA (2001).

5. Gander M.J. ,Halpern L. ,Nataf. F. Optimal convergence for Overlapping and NonOverlapping Schwartz Wave form, relaxation. 11th International Conference on Domain Decomposition Methods, (1999).

6. Billet G., Abgrall R. An adaptive shock capturing algorithm for solvingunsteady reactive flows. Computers and Fluids, 32, pp 1473-1495, (2003). 